PLANETARY NEBULAE IN THE MAGELLANIC CLOUDS

\author{
B. Louise Webster \\ Anglo-Australian Observatory, P.0. Box 296, \\ Epping, N.S.W. 2121, Australia.
}

ABSTRACT

Selection effects in the search for planetary nebulae in the Magellanic Clouds have not seriously distorted the observed space distribution, but have affected the apparent excitation classes. A difference between the properties of the SMC and LMC planetaries remains. A group of high-excitation nebulae with strong lines of $\mathrm{He}$ and $\mathrm{N}$ has been isolated. If these are excluded, the helium abundance appears to be normal in each Cloud. The oxygen abundance in LMC planetaries is about half the Orion value and may be even lower in the SMC objects. The significance of the abundances, excitation classes and space distribution is briefly discussed.

\title{
INTRODUCTION
}

About eighty planetary nebulae have been identified in the Magellanic Clouds (SMC and LMC) and more could be discovered with relative ease. They provide sources of objects at fairly well known distances and in two galaxies that have had quite a different evolutionary history from each other and from the Galaxy. Theories concerning the origin and evolution of galactic planetaries should be able to encompass the properties of the Cloud planetaries. A measurement of the luminosities of Cloud objects with unusual properties can help to clarify their relation to more normal planetaries. Since the planetaries are believed to originate from stars of about 1 to 4 solar masses, information on their chemical abundances and spatial distribution with age contributes to an understanding of the properties of the older population of the Magellanic Clouds. This is required before the formation and evolution of these galaxies can be understood. 
The searches for planetary nebulae in the Magellanic Clouds were reviewed thoroughly by Westerlund (1968) and no major surveys have been published since then. The criteria for the identification of a planetary at the distance of the Clouds have been that it should have an emission spectrum with no detectable continuum on an objective prism plate and that it should be unresolved ( -2 arcsecs) on a high scale direct photograph. The first of these conditions is designed to exclude $\mathrm{Be}$ and other emission-line stars, while the second eliminates small diffuse nebulae, since a resolved nebula would have a gaseous mass greater than about two solar masses. Henize and Westerlund (1963) found that the resolved emission-line objects were all brighter than $\mathrm{M}_{\mathrm{B}}=-3$ and Webster (1969a) demonstrated that since the main contribution to the $B$ magnitude came from the continuum in the resolved objects, the separation in magnitude was a separation between a main sequence or brighter OB star and a sub-luminous hot star. The distinction between diffuse nebulae and planetaries thus seems fairly well established. The condition that the object should have no detectable continuum can, however, exclude low-excitation planetary nebulae. Since such objects generally have strong. [N II] lines near $\mathrm{H} \alpha$ they can be identified by these. For example, in their survey of $\mathrm{H} \alpha$ emission-line stars in the LMC Bohannan and Epps (1974) list eighteen stars with emission at wavelengths other than $\mathrm{H} \alpha$; one of these at least has been confirmed as a planetary by my spectroscopy.

These criteria have been applied fairly rigorously to surveys in the Small Cloud so the only limits to discovery are luminosity and crowding. The known planetaries are concentrated to the central Bar (Westerlund 1968) and their radial velocities, which fall into two velocity groups (Feast 1968), do not correspond to the local H I velocities.

In the Large Cloud the objects listed by Westerlund and Smith (1964) have been subject to some selection in that those with [0 III] 5007 and 4959 less than $H \beta$ on objective prism plates have been discriminated against. It has been known for some time that the apparent distribution of excitation classes differs between the planetaries in the LMC and those in the SMC in the sense that the LMC contains relatively fewer medium to low excitation objects. The selection just mentioned only distorts the distribution of the very lowest classes and is unlikely to account for the excess of very high excitation planetaries in the LMC (Webster 1975).

The second peculiarity about the LMC is that the known planetaries are uniformly distributed over the whole face of the Cloud with no concentration to the Bar where the majority of the low mass stars are located. This has previously been suspected of being a selection effect caused by crowding by background stars. To assess this, objective prism plates covering the central 36 square degrees and over the wavelength range $4550 \AA$ to $5400 \AA$ were obtained for me with the UK 
1.2-m Schmidt, which has a larger plate scale than in the previous surveys. On the least deep of these plates the known planetaries and small $\mathrm{H}$ II regions stand out very clearly and it is estimated that even in the densest part of the Bar less than 20 percent of the bright emission objects would be masked by stars. Unresolved emission-1ine objects ([0 III] $5007,4959, \mathrm{H} \beta$ ) with no continuum were identified to a brightness limit slightly fainter than the Henize (1956) survey, but still well above the plate or crowding limit. Over the field studied these exhibited no concentration either to the Bar or to the regions of active star formation, the distribution being similar to that found previously (and to the 11 known LMC novae).

Smith and Weedman (1972) have discussed the kinematics of the LMC planetaries using velocities measured by themselves, Feast (1968) and Webster $(1969 \mathrm{~b})$. They conclude that the planetary velocities are consistent with the rotation curve defined by the H II regions, but the velocity dispersion around the curve is $15.3 \mathrm{~km} \mathrm{~s}^{-1}$ compared with the $\mathrm{H}$ II value of $7.9 \mathrm{~km} \mathrm{~s}^{-1}$. In the southern part of the cloud beyond the region in which $H$ II regions are found, the dispersion in the planetary velocities increases markedly. In its distribution and kinematics the planetary nebula subsystem appears to be a flattened system, less confined to a plane than the extreme population I component, and with no concentration to the bulk of stars in the Bar.

From a consideration of colour-magnitude diagrams (Tifft and Snel1 1971) and cepheid periods, Payne-Gaposchkin (1974) has concluded that the LMC Bar contains two components, one older than $10^{9}$ years and the other younger than $10^{8}$ years, with relatively little of intermediate age. The distribution of the planetary nebulae (though not necessarily that of the novae) could therefore be understood if the bright planetaries identified at present originated from slightly more massive and younger stars than the bulk of the galactic planetaries.

\section{CHEMICAL ABUNDANCES}

The greatest activity in the subject in the last few years has come from spectral studies designed to determine the physical conditions and chemical abundances in individual objects. Sanduleak et al. (1972) pointed out that the nitrogen lines were systematically weaker in SMC planetaries than in LMC ones, and interpreted this as evidence for a low $\mathrm{N} / \mathrm{H}$ ratio in the SMC objects. The forbidden lines in a nebula are very sensitive to the ionizing radiation and the difference in line strengths could be just a consequence of the excitation-class difference between the Clouds, so detailed studies were clearly required to investigate this. The first major abundance investigation was by Osmer (1976) who made spectroscopic observations of three planetaries in each Cloud and found evidence for a lower $0 / \mathrm{H}$ ratio in the SMC, N/O ratios similar to those of galactic planetaries and an overabundance of helium of 40 percent. He has discussed the relevance of these results very fully. Dufour and Killen (1977) observed three objects, two of 
these in common with Osmer, and in reasonable agreement. Because of an observational difference on the strength of a faint He I line they do not, however, confirm the overabundance of helium in the LMC objects.

In order to illustrate the relation between this new information and the subjects discussed in the last section, I have reconsidered the data of Osmer and Dufour and Killen together with some new observations I have made with the Robinson-Wampler scanner on the Anglo-Australian Telescope (AAT). In Figure 1 the observations are displayed on a diagram designed to illustrate the relation between oxygen abundance (through the electron temperature dependent [0 III] lines) and the excitation class (Webster 1976). The lines correspond to series of models with solar abundances $(A=1)$ or one-tenth solar $(A=0.1)$. Since the electron temperature in a nebula of very low heavy element abundance is limited by cooling from collisional processes in hydrogen (Hummer and Seaton 1964), objects can only lie significantly below the $A=0.1$ line on the diagram if the $\mathrm{N}_{1}+\mathrm{N}_{2} / 4363$ ratio is reduced by collisional de-excitation. The determination of electron temperature from these lines in the usual way is invalid in this case and large errors in the derived oxygen abundance result. In two of the objects Dufour and Killen's determination of widely different temperatures from the [N II] lines confirms that erroneous [O III] temperatures were found.

The conclusions from this diagram are the following. Among the Cloud planetaries is a significant number of dense, high-excitation nebulae with strong [N II] lines (HN objects); similar objects are fairly rare in the Galaxy. Osmer and Dufour and Killen included several of these in their studies which affected the average chemical abundances they derived. Among the other objects, if there is no collisional de-excitation, there is a tendency for the SMC planetaries to be oxygen deficient relative to the LMC ones. This is most marked among the low excitation classes and it is worth recalling the trend among galactic planetaries for low excitation class and low abundance to go together (Aller and Czyzak 1973). This is thought to arise in the analysis, though the coincidence that both planetary nebulae in globular clusters have very low excitation may be relevant.

Al1 the observations have been re-analyzed by the temperature fluctuation method of Peimbert and Costero (1969) with $\mathrm{t}^{2}=0.035$ and the latest atomic data. The results for He, $\mathrm{N}$ and $\mathrm{O}$ are shown in Table 1 together with an identical analysis of the Cloud $H$ II regions and Orion. If the $\mathrm{HN}$ objects are excluded the $\mathrm{N} / \mathrm{O}$ ratio follows the relation (see Danziger et al. 1977) set by the galactic disc planetaries and the planetary in M15.

The HN objects are found to have a mean He/H ratio by number of 0.14 , accounting in part for the high helium value found by Osmer. There are a few galactic planetaries in which it is well established that the helium lines are stronger than normal and several more have been measured recently on the AAT. Most of these have spectra similar 


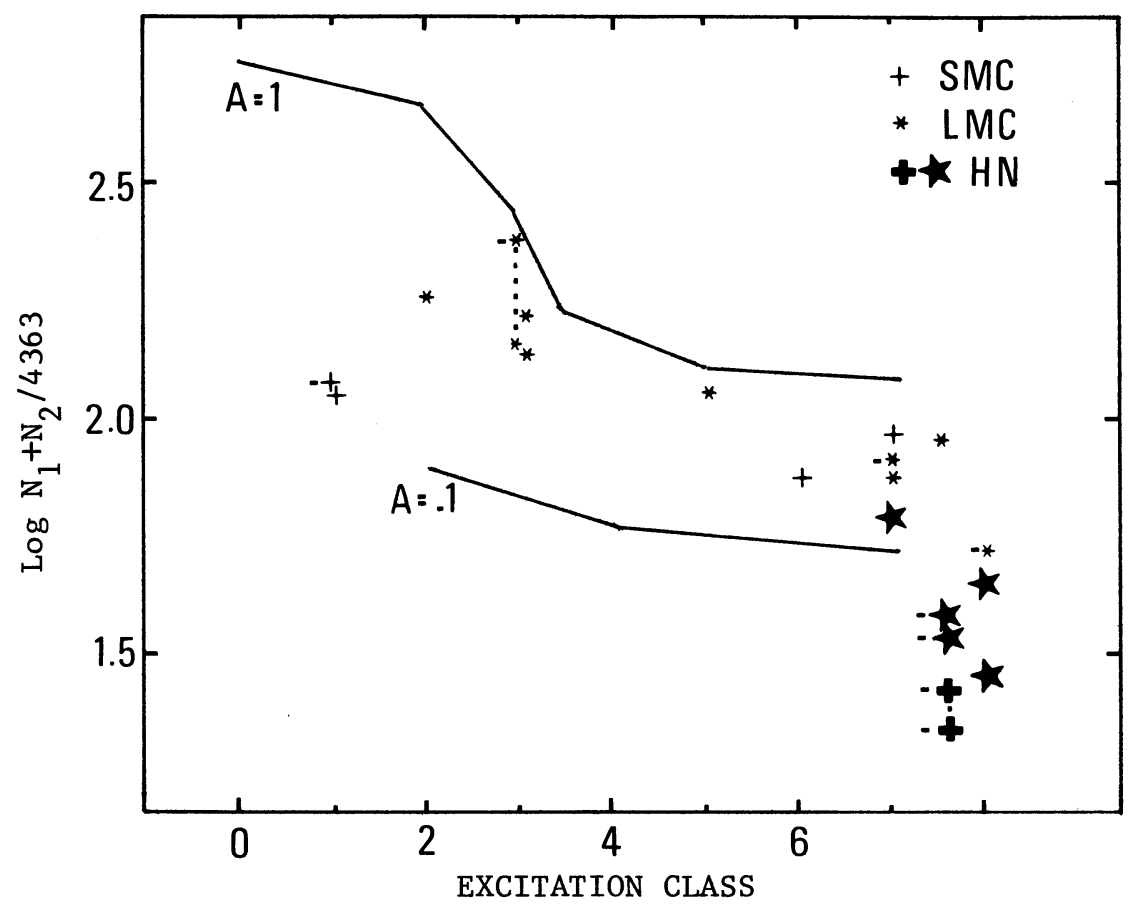

Figure 1. The electron temperature dependent [0 III] lines vs. excitation class diagram for Magellanic Cloud planetaries. Dotted lines join objects measured by two observers and an adjoining dash indicates that the observation was by Osmer or Dufour and Killen. Objects in which [N II] $6584>0.5 \mathrm{Ho}$ are distinguished as $\mathrm{HN}$

Table 1. Average abundances by number relative to hydrogen (excluding HN objects).

\begin{tabular}{lccc}
\hline & \multicolumn{1}{c}{ He } & $0\left(\mathrm{X} \mathrm{10^{4 }}\right)$ & $\mathrm{N}\left(\mathrm{X} \mathrm{10} 0^{5}\right)$ \\
\hline SMC (4 planetaries) & $0.12 \pm .02$ & $2.1 \pm .6$ & 1.2 \\
LMC (10 planetaries) & $0.09 \pm .01$ & $3.5 \pm .3$ & $3.5 \pm .8$ \\
$\begin{array}{l}\text { SMC H II regions } \\
\text { (Dufour 1975) }\end{array}$ & $0.083 \pm .004$ & $1.4 \pm .2$ & $0.5 \pm .1$ \\
$\begin{array}{l}\text { LMC H II regions } \\
\text { (Dufour 1975) }\end{array}$ & $0.084 \pm .004$ & $3.5 \pm .4$ & $1.0 \pm .1$ \\
$\begin{array}{l}\text { Orion } \\
\text { (Peimbert and Torres-Peimbert 1977) }\end{array}$ & 5.62 & 5.75 \\
$\begin{array}{l}\text { Solar } \\
\text { (Lambert 1977) }\end{array}$ & 0.10 & 7.94 & 9.77
\end{tabular}


to the Cloud group and it seems reasonable that the apparent overabundance of helium is related to the class of object rather than to their being located in the Magellanic Clouds.

Although the implication remains that the SMC objects are heavy element deficient relative to those in the LMC, it is worth emphasizing that a real range in abundance would be expected and is observed in the LMC. Close to the red globular cluster NGC 1852 is a planetary nebula that may be a member of it (Webster 1977) in which the nitrogen is between 10 and 20 times underabundant and the oxygen about half as deficient. The LMC thus contains an old, metal-poor component containing a planetary just as our Galaxy does.

An interesting by-product of the spectroscopic work has been the discovery by Feast (1968) that one object (LMC P24) has a faint WolfRayet-like nucleus. This is the first proof of what has generally been assumed and occasionally questioned, that there are low-luminosity W-R stars in planetary nebulae.

\section{DISCUSSION}

Work over the last few years has clarified the properties of the planetary nebula subsystem in each Cloud and begun to concentrate on individual objects. In the Small Cloud the objects cluster in the Bar, have generally low excitation and have oxygen abundances about one third that of Orion with some spread. In the Large Cloud the subsystem is less flattened than the young population I, but not concentrated towards the very old population in the Bar. The oxygen abundance is about one half that of Orion, but one very low abundance planetary exists near a red globular cluster. Helium is generally normal in each Cloud.

It is believed that the helium present in a planetary nebula shell is predominantly primordial, while oxygen has the abundance of the interstellar gas from which the parent star was formed and nitrogen may have been enriched by secondary processes during the evolution of the pre-planetary star. In each of the Clouds the mean planetary oxygen abundance is comparable with that of the $H$ II regions in the respective galaxy. This implies that most of the chemical enrichment took place before the progenitors of the bright planetaries were formed. If the bright planetaries come from the more massive stars this result would be expected, but if they cover the whole mass range then we should conclude that the LMC in particular underwent a very active time of star formation and element synthesis early in its history, just as is believed to have occurred in our Galaxy. The nitrogen abundances are uncertain, but the indication is that they are higher than in the H II regions and that nitrogen has probably been enriched in the star. The HN nebulae in each Cloud are too rare for the planetaries to be major contributors to He and $\mathrm{N}$ enrichment in these galaxies (Osmer 1976). 
In these high excitation nebulae, with very hot nuclei, the high helium and nitrogen abundances must certainly be attributable to enhancement through the CNO cycle. The presence of the nucleosynthesis products in the shell could arise in one of two ways. A greater proportion of the mass of the star could be contained in the shell so that the deeper enriched regions of the envelope have been ejected. This would provide an explanation of the high temperature of the central star since less hydrogen and helium would remain in its outer layers. Alternatively, Aller and Czyzak (1973) have suggested that nitrogenrich planetaries may come from stars more massive than the average planetary since stellar models indicate that in more massive stars deep convective zones can $\mathrm{mix}$ He and $\mathrm{N}$ to the surface. If substantiated this may associate the unexpected spatial distribution of planetaries in the LMC with the excitation class difference.

\section{REFERENCES}

Aller, L.H. \& Czyzak, S.J., 1973. Mem. Soc. Roy. Sci. Liège, V, 285. Bohannan, B. \& Epps, H.W., 1974. Astron. Astrophys., 18, 47.

Danziger, I.J., Dopita, M.A., Hawarden, T.G.\&Webster, B.L., 1977. preprint.

Dufour, R.J., 1975. Astrophys. J., 195, 315.

Dufour, R.J. \& Killen, R.M., 1977. Astrophys. J., 211, 68.

Feast, M.W., 1968. Mon. Not. Roy. astr. Soc., 140, 345 .

Henize K.G., 1956. Astrophys. J. Supp1., 2, 315.

Henize, K.G. \& Westerlund, B.E., 1963. Astrophys. J., 137, 747.

Hummer, D.G. \& Seaton, M.J., 1964. Mon. Not. Roy. astr. Soc., 127, 217.

Lambert, D.L., 1968. Mon. Not. Roy. astr. Soc., 138, 143.

Osmer, P.S., 1976. Astrophys. J., 203, 352.

Payne-Gaposchkin, C.H., 1974. Smithsonian Contr. 16.

Peimbert, M. \& Torres-Peimbert, S., 1977. Mon. Not. Roy. astr. Soc., $179,217$.

Peimbert, M. \& Costero, R., 1969. Bol. Ton. y Tac., 5, 3.

Sanduleak, N., MacConne11, D.J. \& Hoover, P., 1972. Nature, 237, 28.

Smith, M.G. \& Weedman, D.W., 1972. Astrophys. J., 177, 595.

Tifft, W.G. \& Snell, C.M., 1971. Mon. Not. Roy. astr. Soc., 151, 365.

Webster, B.L., 1969a. Mon. Not. Roy. astr. Soc., 143, 79.

Webster, B.L., 1969b. Mon. Not. Roy. astr. Soc., $\overline{143}, 97$.

Webster, B.L., 1975. Mon. Not. Roy. astr. Soc., $\frac{173}{17}, 437$.

Webster, B.L., 1976. Mon. Not. Roy. astr. Soc., 174, 513.

Webster, B.L., 1977. Pub1. astron. Soc. Pac., 88, 669.

Westerlund, B.E., 1968. I.A.U. Symp. No. 34, 23.

Westerlund, B.E. \& Smith, L.F., 1964. Mon. Not. Roy. astr. Soc., 127,449 . 


\section{DISCUSSION}

Aller: Are not the objects with large $\mathrm{He}$ and $\mathrm{N}$ abundances systematically bright? That is, one tends to pick intrinsically bright, highmass objects - whereas in our galaxy we get a range of objects. Webster: Yes. I think that it is just that selection that accounts for the unexpected excitation class and spatial distribution of the planetary nebulae in the LMC.

Peimbert, M.: What is the main sequence turnoff of the red globular cluster in the LMC where the new planetary nebulae was found? Webster: The only information relevant to the age of the cluster is its integrated colors, which are quite red. We can only say that it belongs to the older group of globular clusters in the LMC.

Seaton: Are there intrinsic differences between Cloud and Galactic planetary nebulae, which may invalidate the attempt to use results for the Clouds to calibrate the Galactic planetary nebulae distance scale? Webster: The method for determining the planetary distance scale from the Clouds that we both used in 1968-9 must be affected by the mass distribution of the parent stars in the Clouds relative to those in the Glaaxy. However, we probably select the most massive shells in each case so that the calibration would be valid for these. The possibility of obtaining a luminosity function of the planetaries to fainter limits in the future should considerably improve the calibration.

Moseley: Could the lack of concentration of planetaries to the bar in the LMC be due to extinction in the bar?

Webster: Small clouds of dust are clearly seen projected against the

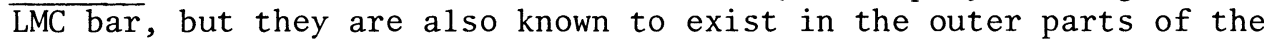
galaxy. One might expect the dust to roughly follow the distribution of the interstellar gas which is not concentrated to the bar, and so I do not feel that interstellar extinction would seriously distort the observed space distribution of planetaries.

Peimbert, M.: The results mentioned by Dr. Webster on the existence of He-N rich planetary nebulae in the Magellanic Clouds are of great importance. These objects belong to a distinct type of planetary nebulae. In the Galaxy there are seven out of about fifty well observed objects that belong to this type.

Dopita: I have found several $\mathrm{N}$ rich planetaries in my search for new Herbig-Haro objects. These were selected on morphological criteria, a filamentary structure and apparent association with dark lanes. Have you any comment on why such a characteristic structure should be specific to this class; and have you seen any central stellar object in the planetaries you have studied?

Webster: My only comment about why the filamentary structure should exist is that in one object quite high expansion velocities are measured. I have not observed a central star in any of them. 